\title{
Adsorption on graphene: flat to edge to end transitions of phenyl hydroquinone
}

\author{
Lifu Chen, Eden E. L. Tanner and Richard G. Compton* \\ Department of Chemistry, Physical and Theoretical Chemistry Laboratory, Oxford University, \\ South Parks Road, Oxford OX1 3QZ, UK
}

"To whom correspondence should be addressed

Email: richard.compton@chem.ox.ac.uk

Phone: +44 (0) $1865275957 \quad$ Fax: +44 (0) 1865275410

Keywords:

Graphene, nanomaterials, phase transition, nano-impacts, adsorption

To be submitted to:

Physical Chemistry Chemical Physics (PCCP) 


\section{Abstract}

The adsorption of phenyl hydroquinone (PHQ) of graphene surfaces at the liquid-solid interface is investigated revealing a flat orientation and two different vertically adsorbed states of PHQ on graphene nanoplatelets (GNPs), namely edgewise or endwise adsorption. The transition between these states is driven by increasing concentrations of PHQ in solution leading to increased absolute coverages on the graphene surface. At low adsorbate concentrations $(\leq 21 \mathrm{mM})$, the adsorption process is also shown to be Langmuirian with an adsorption constant of $(9.5 \pm 0.2) \mathrm{mM}^{-1}$. Independent measurements are conducted using a single particle electrochemical technique to confirm the surface coverage of PHQ on GNPs at low concentrations, showing excellent agreement with the UV-Vis studies. 
Since its discovery, graphene ${ }^{1}$ has been extensively studied. The high thermal and electronic conductivities, large surface area, high charge and carrier mobility and strong mechanical strength encourage the application of graphene and its derivatives in a variety of contexts, from electronics to medicine. ${ }^{2-20}$ Two particularly interesting branches are the production of new and advanced catalytic materials, ${ }^{18}$ where graphene features extensively both as catalyst support and as catalyst itself, and the development of ultra-sensitive graphene-based chemical detectors ${ }^{8}$ and biological molecule sensors, ${ }^{20}$ which are capable of detecting individual molecules adsorbed on graphene. Accordingly, it is of fundamental importance to quantify and understand the behaviour of molecules on graphene surfaces.

Hitherto, the adsorption of metal atoms, ${ }^{21}$ small gas molecules, ${ }^{22}$ halogens ${ }^{23,}{ }^{24}$ and small organic molecules ${ }^{25,26}$ on graphene have been widely studied. However, little is known about the adsorption of more complex, larger, especially organic, molecules on graphene, especially with respect to their orientation at the graphene-water interface. In contrast, the adsorption of large organic molecules at metal surfaces has been intensely investigated theoretically ${ }^{27,28}$ and experimentally ${ }^{29}, 30$ in the recent decades. In particular, the orientation of adsorbed organic molecules on a platinum surface in aqueous solution has been extensively studied by Hubbard and co-workers, including a selection of forty aromatic compounds. ${ }^{31-34}$ Mostly, aromatic compounds adsorb in a horizontal orientation, with the aromatic system parallel to the Pt surface at low adsorbate concentrations. At higher concentrations, some organic molecules reorient to take a more vertical orientation with the aromatic ring perpendicular to the surface. Our recent work on graphene $e^{35,36}$ has focused on the adsorption and desorption of organic molecules on individual graphene nanoplatelets (GNPs), a graphene derivative which has the advantageous properties of graphene, but circumvents the poor stability traditionally experienced. ${ }^{19,} 37$ Specifically, adsorption of 1,2-dihydroxybenzene (catechol) on the GNPs was shown to be reversible and at low concentrations to follow the Langmuir 
isotherm. At higher concentrations the adsorbate is inferred to undergo a flat to vertical concentration driven phase transition. To seek new insights into such adsorption processes, we here consider the possibility of two different vertically adsorbed states reflecting the edge or end adsorption of a 'box' shaped molecule. In particular, we focus on the adsorption of phenyl hydroquinone (PHQ) on GNPs from aqueous solutions. A wide range of concentrations of PHQ solution are used to modify the GNPs. The uptake of PHQ is followed by UV-Vis spectroscopy - hence allowing the accurate amount of PHQ molecules adsorbed by GNPs to be determined. The adsorption isotherm then can be constructed and three plateaux are observed, indicating that three molecular orientation states of PHQ on GNPs exist. By comparing the area occupied by each individual PHQ molecule on GNPs, corresponding to each plateau, with the theoretical molecular area of different orientations, the orientation states of PHQ on GNPs can be determined. To confirm this orientation state and enable single PHQ-GNPs measurements, the nanoimpact method ${ }^{38}$ is applied. In this method, a suspension of nanoparticles move by virtue of their Brownian motion which undergo stochastic collisions with a potentiostatted microelectrode, resulting in a 'spike' in the current that corresponds to the Faradaic electron transfer of the adsorbed molecules. The spike in current has been shown to directly relate to the amount of material undergoing

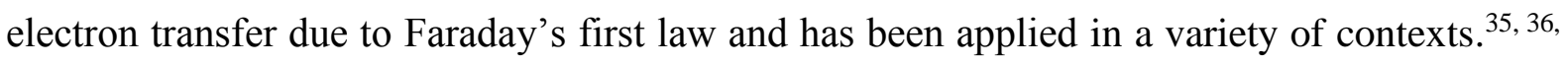
38, 39 The surface coverage of electroactive species (PHQ) on GNPs could then be obtained based on the charge passed per individual impact, allowing the molecular orientation of PHQ to be inferred.

First, to maximise the solubility of PHQ in aqueous solution, a buffered solution containing 19.75 M methanol and 0.2 M HCl was chosen (See SI for full experimental details). Buffered solutions containing a range of PHQ concentration were then prepared and examined by UVVis spectroscopy, enabling the construction of a calibration curve between PHQ 
concentration and absorbance. A linear region was obtained, as shown in Figure S1. Next, the GNPs were modified by mixing $1 \mathrm{~mL}$ of a known concentration of PHQ in buffered solution with GNPs. They were then sonicated for 35 min to allow maximum adsorption, followed by centrifugation. The absorbance of the original PHQ solution before adsorption and the supernatant after adsorption were both measured by UV-Vis spectroscopy. As shown in Figure S2, the absorbance of phenyl hydroquinone in aqueous solution reaches a maximum at $301 \mathrm{~nm}$, consistent with literature. ${ }^{40}$ An obvious reduction in magnitude of the absorbance peak can be observed, due to the adsorption onto the GNPs, allowing the adsorbed amount of PHQ by GNPs to be determined. These results were then used to plot the adsorption isotherm of PHQ for GNPs in buffed solution, as shown in Figure 1a, where three clear plateaux can be observed. A semi-logarithmic plot was also constructed (Figure 1b) to more clearly examine the first plateau.
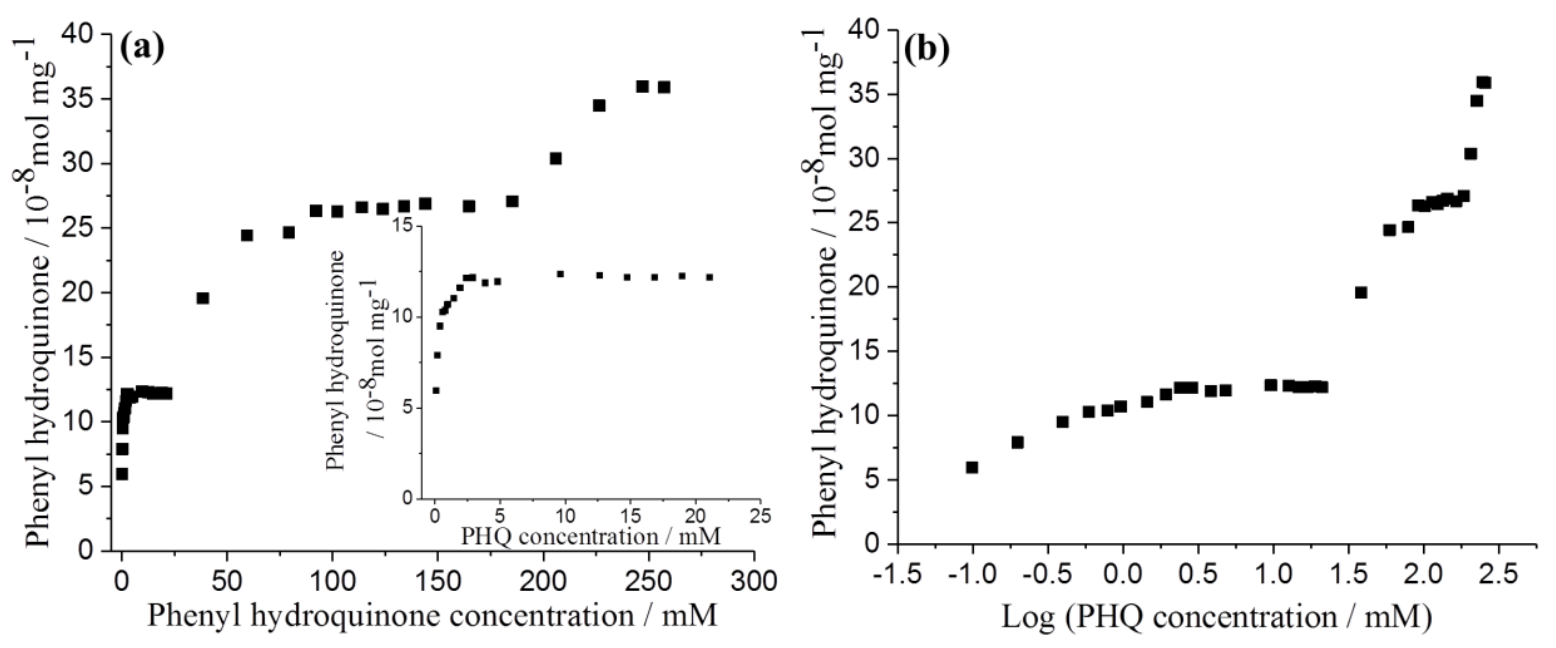

Figure 1. (a) Phenyl hydroquinone adsorption isotherm for GNPs in buffered solution (19.75 $\mathrm{M}$ methanol and $0.2 \mathrm{M} \mathrm{HCl}$ ). Inset: zoom-in of PHQ adsorption isotherm for GNPs when concentration below than $21 \mathrm{mM}$. (b) Log PHQ concentration plot.

With increasing of PHQ concentration, the amount of PHQ adsorbed by a unit amount $(1 \mathrm{mg})$ of GNPs (n) increases progressively and reaches the first plateau at a PHQ concentration between ca. 2 and $21 \mathrm{mM}$ with $n_{\max }=1.2 \times 10^{-7} \mathrm{~mol} \mathrm{mg}^{-1}$, giving the maximum surface coverage $\left(\Gamma_{\max }\right)$ of $(1.9 \pm 0.6) \times 10^{-10} \mathrm{~mol} \mathrm{~cm}^{-2}$ (see SI for detailed calculations). The second 
plateau is reached at an adsorbate concentration of $c a .92$ to $185 \mathrm{mM}$ with $n_{\max }{ }^{\prime}=2.7 \times 10^{-7}$ mol $\mathrm{mg}^{-1}$ and $\Gamma_{\max }{ }^{\prime}=(4.3 \pm 1.5) \times 10^{-10} \mathrm{~mol} \mathrm{~cm}^{-2}$, whilst the third plateau occurs at concentrations between $c a .240$ and $260 \mathrm{mM}$ with $n_{\max } "=3.6 \times 10^{-7} \mathrm{~mol} \mathrm{mg}^{-1}$, giving $\Gamma_{\max }$ " $=(5.8 \pm 1.9) \times 10^{-10} \mathrm{~mol} \mathrm{~cm}^{-2}$. The increasing $\Gamma$ at higher adsorbate concentrations is inferred to result from a molecular phase transition to a more densely packed layer corresponding to a different orientation rather than multilayer adsorption, as the coverage for both catechol ${ }^{36}$ and PHQ eventually plateaus, that the coverages for the different orientations are consistent with the known molecular geometry. That said the possible formation of multilayers cannot be completely ruled out.

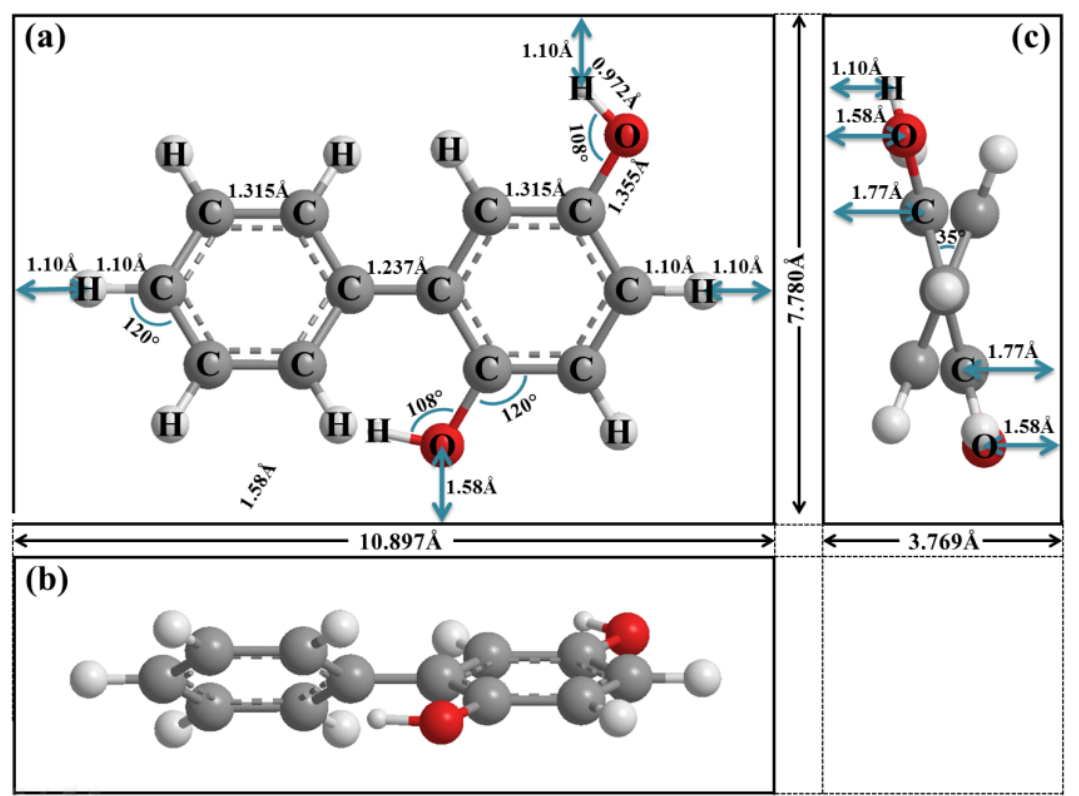

Scheme 1. Rectangular box model of phenyl hydroquinone molecule for both (a) flat view, (b) edgewise view and (c) endwise view

In order to confirm the molecular orientation, it is necessary to obtain the theoretical area of the molecule in each possible orientation. This can be achieved by approximating the PHQ molecule as a rectangular box with all side lengths estimated by trigonometry for bond lengths, bond angles and Van de Waals radii of terminating atoms (Scheme 1). The data for bond lengths, bond angles were obtained from ChemDraw 15.1 and Van de Waals radii of 
terminating atoms tabulated by Rowland were used. ${ }^{41} S_{\mathrm{PHQ}}$ of flat view, $S_{\mathrm{PHQ}}$ ' of edgewise view, and $S_{\mathrm{PHQ}}$ " of endwise view hence can be estimated as $8.5 \times 10^{-15} \mathrm{~cm}^{2}, 4.1 \times 10^{-15} \mathrm{~cm}^{2}$ and $2.9 \times 10^{-15} \mathrm{~cm}^{2}$ respectively, which are in good agreement with literature. ${ }^{31,34}$ The average area occupied by each individual PHQ molecule ( $\left.S_{\mathrm{R}-\mathrm{PHQ}}\right)$ on GNPs can be obtained from the surface coverage, $\Gamma$, via $S_{\mathrm{R}-\mathrm{PHQ}}=1 /\left(N_{\mathrm{A}} \Gamma\right)$, where $N_{\mathrm{A}}$ is the Avogadro constant. Therefore, $S_{\mathrm{R}-\mathrm{PHQ}}$ can be determined as $(8.5 \pm 2.1) \times 10^{-15} \mathrm{~cm}^{2}$ at the first plateau, $(3.8 \pm 0.9)$ $\times 10^{-15}$ at the second plateau and $(2.9 \pm 0.7) \times 10^{-15}$ at the third plateau, consistent with the flat PHQ molecule area of $8.5 \times 10^{-15} \mathrm{~cm}^{2}$ (Scheme 1a), edgewise PHQ molecule area of 4.1 $\times 10^{-15} \mathrm{~cm}^{2}$ (Scheme 1b) and endwise PHQ molecule area of $2.9 \times 10^{-15} \mathrm{~cm}^{2}$ (Scheme 1c), respectively. This gives new physical insight into the orientation states and phase transitions of large organic molecules adsorbed on graphene. The PHQ molecules adopt a flat orientation adsorbed on the GNPs surface at low concentration $(\leq 21 \mathrm{mM})$, whilst they adopt an edgewise orientation at medium concentrations $(92<\mathrm{C}<185 \mathrm{mM})$. At intermediate concentrations, a flat to edgewise phase transition is inferred to occur, but it remains an open question if the different oriented molecules are locally organised or randomly distributed. Similarly, PHQ molecules adopt an endwise orientation at high concentrations $(\geq 240 \mathrm{mM})$ and an edgewise to endwise phase transition is inferred to take place when the adsorbate concentration increases from a medium concentration region to a high concentration region. 


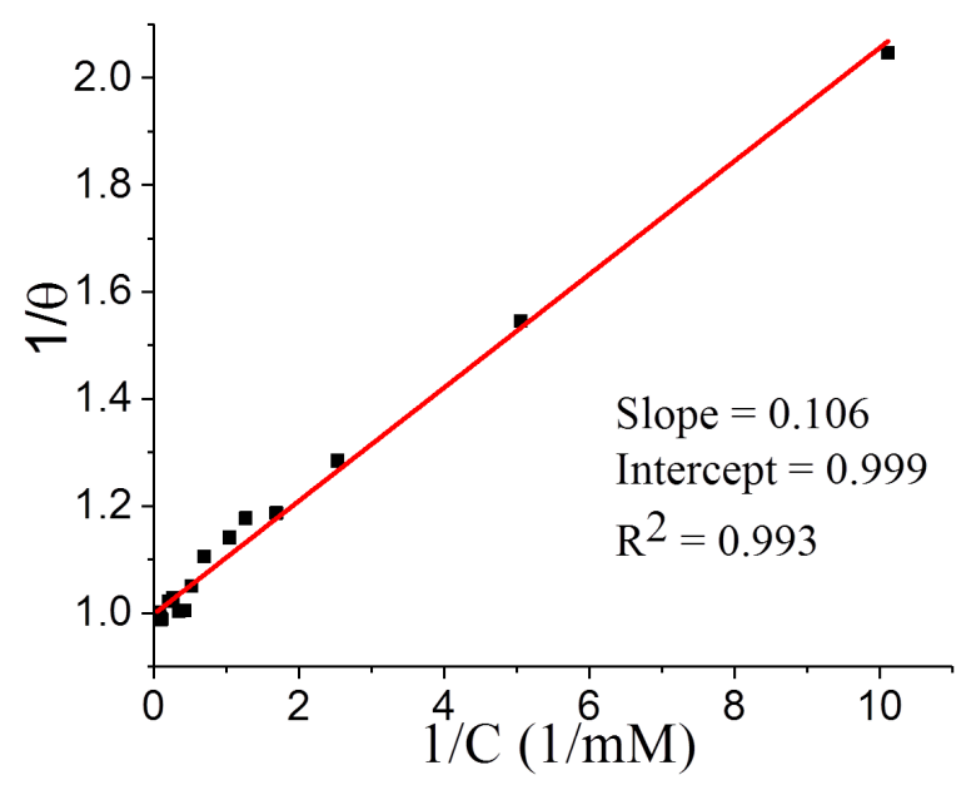

Figure 2. Langmuir plot of PHQ on GNPs in buffered solution (19.75 M methanol and $0.2 \mathrm{M}$ $\mathrm{HCl}$ ), where $\Theta$ is the fractional surface coverage and $\mathrm{C}$ is the adsorbate concentration, Langmuir adsorption model applies when the PHQ concentration is lower than $21 \mathrm{mM}$.

Last, the low concentration region below the onset of the second plateau $(\leq 21 \mathrm{mM})$ was analysed in terms of the Langmuir model, which predicts the fractional coverage, $\Theta$, to vary with adsorbate concentration, $\mathrm{C}$,

$\theta=\frac{\mathrm{KC}}{1+\mathrm{KC}}$

where $\Theta=\Gamma / \Gamma_{\max }$ and $\Gamma$ is the coverage corresponding to the adsorbate concentration. As shown in Figure 2, 1/ $\Theta$ was plotted against 1/C, giving a straight line with intercept of 0.999 and $\mathrm{R}^{2}=0.993$. This indicates that the adsorption of PHQ onto GNPs before the phase transition is in a good agreement with the Langmuir model. Meanwhile, the adsorption constant $(\mathrm{K})$ can be determined from the reciprocal of the slope, which is 0.106 , giving $(9.5 \pm$ 0.2) $\mathrm{mM}^{-1}$. The success of the Langmuir model also suggests that the adsorption for PHQ onto GNPs is reversible. 


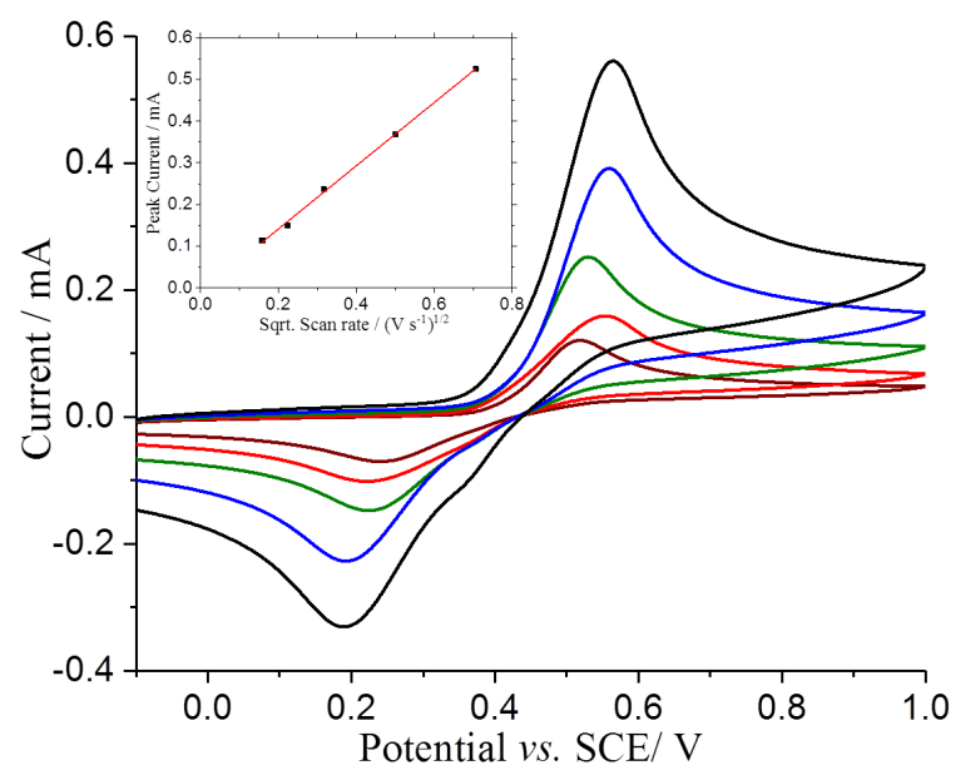

Figure 3. Cyclic voltammograms of $3 \mathrm{mM}$ PHQ in buffered solution (19.75 $\mathrm{M}$ methanol and $0.2 \mathrm{M} \mathrm{HCl}$ ) on a BPPG electrode at scan rate of $25 \mathrm{mV} \mathrm{s}^{-1}$ (brown), $50 \mathrm{mV} \mathrm{s}^{-1}$ (red), $100 \mathrm{mV}$ $\mathrm{s}^{-1}$ (green), $250 \mathrm{mV} \mathrm{s}^{-1}$ (blue) and $500 \mathrm{mV} \mathrm{s}^{-1}$ (black). Inset: Oxidative peak current as a function of the square root of scan rate. A red line indicates the best-fit line.

Having evidenced the adsorption of PHQ on the GNPs spectroscopically, an electrochemical method was then used to confirm the surface coverage of PHQ on GNPs and the orientation in the horizontal state. To determine the approximate oxidation potentials required for subsequent nano-impact experiments, cyclic voltammetry (CV) was conducted using a basalplane pyrolytic graphite (BPPG) electrode. Cyclic voltammograms were recorded on a bare BPPG macroelectrode in a buffered solution (19.75 M methanol and $0.2 \mathrm{M} \mathrm{HCl})$ containing 3 $\mathrm{mM}$ PHQ and saturated with nitrogen by sweeping the potential from -0.10 to $+1.00 \mathrm{~V} v s$. SCE and reversed back to $-0.20 \mathrm{~V}$ at a scan rate of $25 \mathrm{mV} \mathrm{s}^{-1}$ (Figure 3, brown line). The resultant voltammogram showed an single oxidative peak at $c a .+0.52 \mathrm{~V}$ vs. SCE and a reductive peak at $c a .+0.24 \mathrm{~V} v s$. SCE. The peak current is also found to be proportional with the square root of scan rate (Figure 3 inset) indicating that it is a diffusion controlled electrochemical process. 


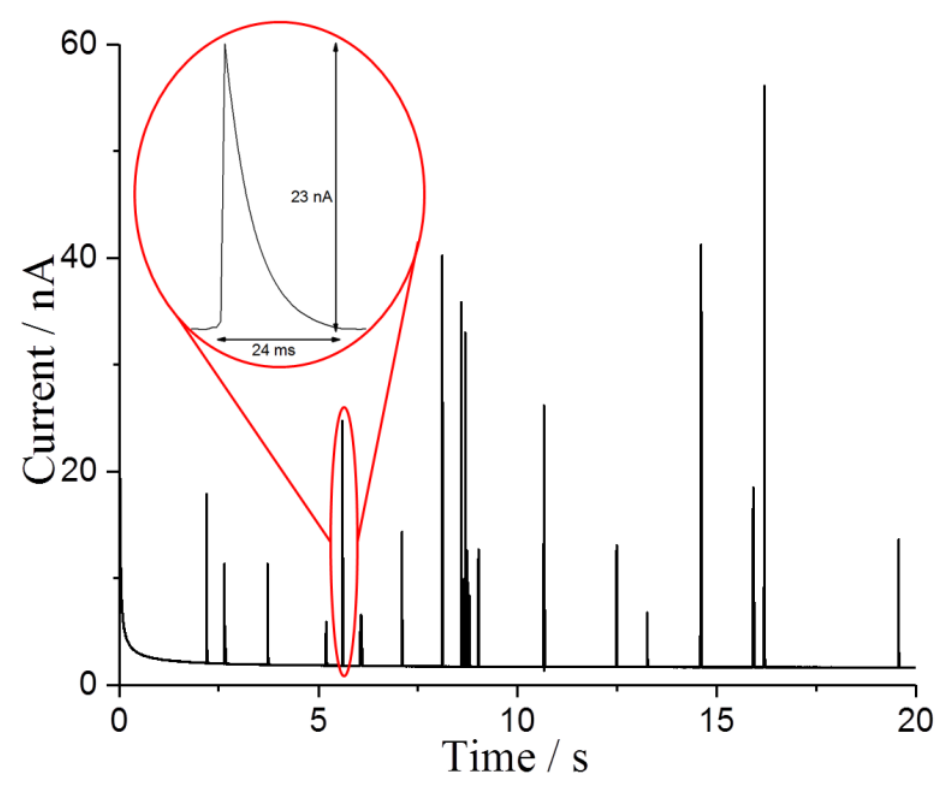

Figure 4. Representative chronoamperometric profiles of a $3.33 \times 10^{-14} \mathrm{M}$ suspension of PHQ-GNPs containing $19.75 \mathrm{M}$ methanol and $0.2 \mathrm{M} \mathrm{HCl}$ buffered solution at $+1.0 \mathrm{~V} v s$. SCE. Next, a clean cylindrical carbon fibre wire microelectrode (of length $c a .1 \mathrm{~mm}$ and diameter ca. $7 \mu \mathrm{m})$ was first immersed in buffered solution and a known concentration $(0.33 \mathrm{pM})$ of dispersed PHQ-GNPs suspension was added. The oxidative potential used for nano-impact experiments was $+1.0 \mathrm{~V} v s$. SCE, a potential value more positive than our initial results chosen to ensure that the complete oxidation of PHQ would occur, as previous studies have shown that at low overpotentials an incomplete oxidation may occur. ${ }^{35,} 39$ Under potentiostatted conditions, +1.0 V vs. SCE, clear and sharp oxidative spikes with a short duration (10-25 ms) from individual PHQ-GNPs were observed as shown in Figure 4. For comparison, a control experiment was conducted in the same conditions without addition of PHQ-GNPs suspension (Figure S3). No spikes were detected confirming that each individual spike results from the random collisions between the single PHQ-GNPs and the electrode, and subsequent two-electron, two-proton oxidation of $\mathrm{PHQ}^{42}$ and electron transfer to the electrode (Scheme 2). 


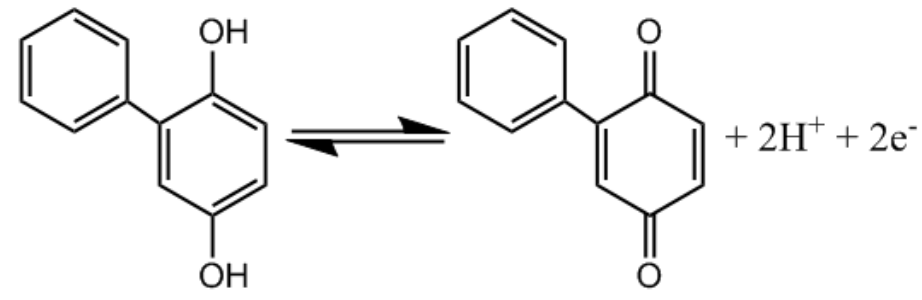

Scheme 2. Two-electron, two-proton oxidation of adsorbed PHQ

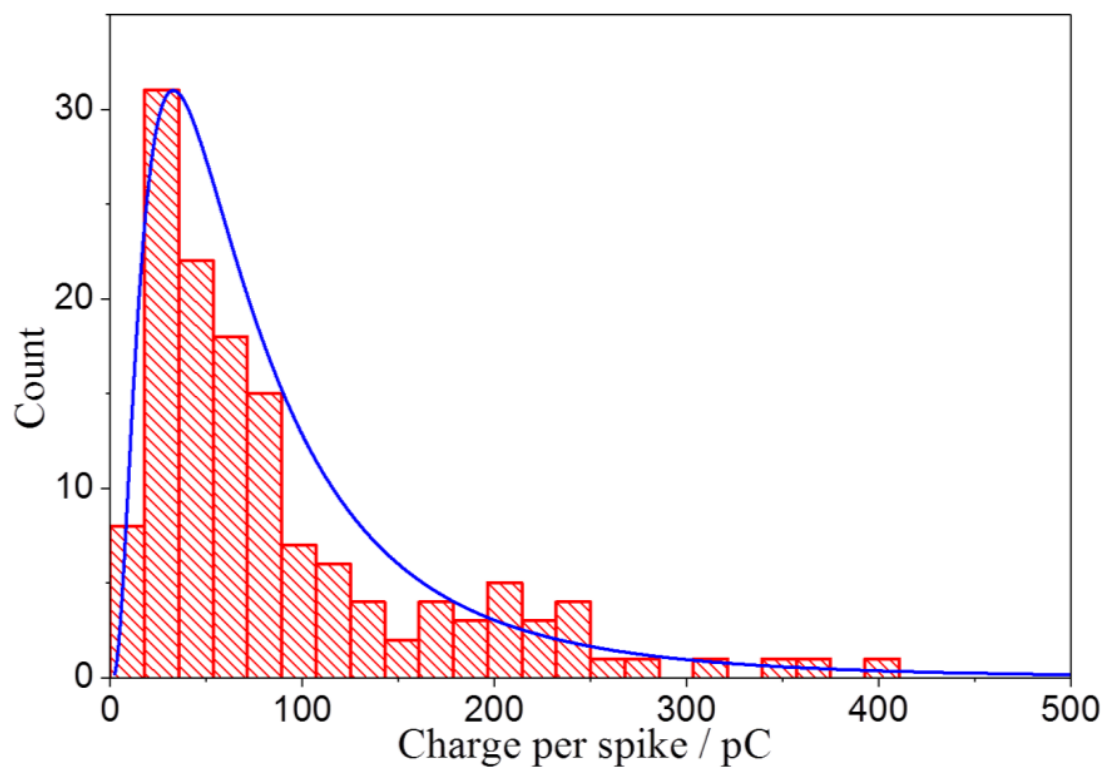

Figure 5. Distribution of charge from oxidation of single PHQ-GNPs by analysis of the 146 current spikes at $+1.0 \mathrm{~V} v s$. SCE. The log-normal distribution is plotted in the blue curve.

A total of 146 single spikes were detected from ten 20 s chronoamperometric scans. The charge passed per spike $(Q)$ was then quantified by integrating the area underneath the individual spikes. Figure 5 shows the distribution of charge of spikes only with a single-peak, sharp shape. This distribution reflects the range of size of GNP present. ${ }^{43}$ The multi-peak spikes might results from collision between multi PHQ-GNPs and the electrode simultaneously, and hence were not included in the analysis. The duration of impacts were analysed in Figure S4. The average charge was determined to be $(94.4 \pm 7.4) \mathrm{pC}$. The error bar was derived from $\mathrm{SD} /(n)^{1 / 2}$, where $\mathrm{SD}$ is the standard deviation and $n$ is the number of the spikes. 
If the charge passed $(Q)$ is as a result of complete oxidation of PHQ on single GNPs as the simultaneous capacitative charge transfer caused by the GNPs is negligible, ${ }^{35}$ then $Q$ can be related to the number of PHQ molecules adsorbed per GNP $(N)$ via $Q=n N e$, where $n$ is the number of electrons transferred during oxidation of PHQ $(n=2)$, and $e$ is the charge per electron. The surface coverage $(I)$ and the area occupied by each individual PHQ molecule $\left(S_{\mathrm{R}-\mathrm{PHQ}}\right)$ on the GNP can be determined as $(1.6 \pm 0.6) \times 10^{-10} \mathrm{~mol} \mathrm{~cm}^{-2}$ and $(10.1 \pm 4.1) \times 10^{-}$ ${ }^{15} \mathrm{~cm}^{2}$ per molecule, respectively (see SI for detailed calculations). Comparing $\Gamma$ of GNP modified by $3 \mathrm{mM}$ PHQ buffed solution from nanoimpact method, which is $(1.6 \pm 0.6) \times 10^{-10}$ mol cm$~^{-2}$, with $\Gamma_{\max }$ at the first plateau obtained from UV-Vis with value of $(1.9 \pm 0.6) \times 10^{-10}$ mol cm$~^{-2}$ shows an excellent agreement and suggests a flat orientation of PHQ molecules on GNPs at this concentration. The nanoimpact method is most useful at low PHQ concentrations. At higher concentrations, the excess modifier molecules introduce an extremely high background current leading to no spikes being detected. Previous work ${ }^{36}$ applied a wash-step to remove excess modifier molecules to investigate GNPs modified with higher adsorbate concentrations. However, the reversible Langmuirian adsorption results in an inevitable desorption process which occurs during the washing leading to an underestimated surface coverage at high concentrations.

In conclusion, the present method allows the accurate measurements of the amount of large organic molecules adsorbed on GNPs, hence probing the orientation states and concentration driven phase transition of adsorbed molecules on the adsorbent. The adsorption of PHQ on GNPs is reversible and, at low concentrations Langmuirian. With the increase of adsorbate concentrations, first a flat to edgewise then an edgewise to endwise phase transition change is inferred. 


\section{Acknowledgements}

The research leading to these results has received partial funding from the European Research

Council under the European Union's Seventh Framework Programme (FP/2007-2013)/ERC

Grant Agreement no. [320403].

\section{References}

1. K. S. Novoselov, A. K. Geim, S. V. Morozov, D. Jiang, Y. Zhang, S. V. Dubonos, I. V. Grigorieva and A. A. Firsov, Science (New York, N.Y.), 2004, 306, 666-669.

2. K. N. Kudin, B. Ozbas, H. C. Schniepp, R. K. Prud'homme, I. A. Aksay and R. Car, Nano letters, 2008, 8, 36-41.

3. S. V. Morozov, K. S. Novoselov, M. I. Katsnelson, F. Schedin, D. C. Elias, J. A. Jaszczak and A. K. Geim, Physical Review Letters, 2008, 100, 016602.

4. C.-H. Park, F. Giustino, C. D. Spataru, M. L. Cohen and S. G. Louie, Nano letters, 2009, 9, 4234-4239.

5. A. Ambrosi, C. K. Chua, A. Bonanni and M. Pumera, Chemical Reviews, 2014, 114, 7150-7188.

6. S. Stankovich, D. A. Dikin, G. H. B. Dommett, K. M. Kohlhaas, E. J. Zimney, E. A. Stach, R. D. Piner, S. T. Nguyen and R. S. Ruoff, Nature, 2006, 442, 282-286.

7. A. K. Geim and K. S. Novoselov, Nature Materials, 2007, 6, 183-191.

8. F. Schedin, A. K. Geim, S. V. Morozov, E. W. Hill, P. Blake, M. I. Katsnelson and K. S. Novoselov, Nature Materials, 2007, 6, 652-655.

9. D. A. Dikin, S. Stankovich, E. J. Zimney, R. D. Piner, G. H. B. Dommett, G. Evmenenko, S. T. Nguyen and R. S. Ruoff, Nature, 2007, 448, 457-460.

10. A. A. Balandin, S. Ghosh, W. Bao, I. Calizo, D. Teweldebrhan, F. Miao and C. N. Lau, Nano letters, 2008, 8, 902-907.

11. D. Li, M. B. Müller, S. Gilje, R. B. Kaner and G. G. Wallace, Nature Nanotechnology, 2008, 3, 101-105.

12. A. H. Castro Neto, F. Guinea, N. M. R. Peres, K. S. Novoselov and A. K. Geim, Reviews of Modern Physics, 2009, 81, 109-162.

13. K. S. Kim, Y. Zhao, H. Jang, S. Y. Lee, J. M. Kim, K. S. Kim, J. H. Ahn, P. Kim, J. Y. Choi and B. H. Hong, Nature, 2009, 457, 706-710.

14. A. K. Geim, Science (New York, N.Y.), 2009, 324, 1530-1534.

15. D. R. Dreyer, S. Park, C. W. Bielawski and R. S. Ruoff, Chemical Society Reviews, 2010, 39, 228-240.

16. S. Bae, H. Kim, Y. Lee, X. Xu, J. S. Park, Y. Zheng, J. Balakrishnan, T. Lei, H. Ri Kim, Y. I. Song, Y. J. Kim, K. S. Kim, B. Özyilmaz, J. H. Ahn, B. H. Hong and S. Iijima, Nature Nanotechnology, 2010, 5, 574-578.

17. F. Schwierz, Nature Nanotechnology, 2010, 5, 487-496.

18. Y. Liang, Y. Li, H. Wang, J. Zhou, J. Wang, T. Regier and H. Dai, Nature Materials, 2011, 10, 780-786. 
19. K. S. Novoselov, V. I. Fal'Ko, L. Colombo, P. R. Gellert, M. G. Schwab and K. Kim, Nature, 2012, 490, 192-200.

20. A. Bonanni, C. K. Chua, G. Zhao, Z. Sofer and M. Pumera, ACS Nano, 2012, 6, 85468551.

21. D. Krepel and O. Hod, The Journal of Physical Chemistry C, 2013, 117, 19477-19488.

22. J. Dai, J. Yuan and P. Giannozzi, Applied Physics Letters, 2009, 95, 232105.

23. H. Widjaja, Z. T. Jiang, M. Altarawneh, C. Y. Yin, B. M. Goh, N. Mondinos and B. Z. Dlugogorski, Applied Surface Science, 2015, 356, 370-377.

24. H. Şahin and S. Ciraci, Journal of Physical Chemistry C, 2012, 116, 24075-24083.

25. C. Thierfelder, M. Witte, S. Blankenburg, E. Rauls and W. G. Schmidt, Surface Science, 2011, 605, 746-749.

26. E. Schröder, Journal of Nanomaterials, 2013, 2013.

27. W. Liu, A. Tkatchenko and M. Scheffler, Accounts of Chemical Research, 2014, 47, 3369-3377.

28. R. J. Maurer, V. G. Ruiz, J. Camarillo-Cisneros, W. Liu, N. Ferri, K. Reuter and A. Tkatchenko, Progress in Surface Science, 2016, 91, 72-100.

29. S. M. Barlow and R. Raval, Surface Science Reports, 2003, 50, 201-341.

30. F. Rosei, M. Schunack, Y. Naitoh, P. Jiang, A. Gourdon, E. Laegsgaard, I. Stensgaard, C. Joachim and F. Besenbacher, Progress in Surface Science, 2003, 71, 95-146.

31. M. P. Soriaga and A. T. Hubbard, Journal of the American Chemical Society, 1982, 104, 2735-2742.

32. M. P. Soriaga and A. T. Hubbard, Journal of the American Chemical Society, 1982, 104, 2742-2747.

33. M. P. Soriaga and A. T. Hubbard, Journal of the American Chemical Society, 1982, 104, 3937-3945.

34. M. P. Soriaga, P. H. Wilson, A. T. Hubbard and C. S. Benton, Journal of Electroanalytical Chemistry and Interfacial Electrochemistry, 1982, 142, 317-336.

35. H. Wu, Q. Lin, C. Batchelor-McAuley, L. M. Goncalves, C. F. Lima and R. G. Compton, The Analyst, 2016, 141, 2696-2703.

36. L. Chen, X. Li, E. E. L. Tanner and R. G. Compton, Chemical Science, 2017, DOI: 10.1039/C7SC01331K.

37. L. Liu, S. Ryu, M. R. Tomasik, E. Stolyarova, N. Jung, M. S. Hybertsen, M. L. Steigerwald, L. E. Brus and G. W. Flynn, Nano letters, 2008, 8, 1965-1970.

38. W. Cheng and R. G. Compton, TrAC Trends in Analytical Chemistry, 2014, 58, 79-89.

39. Q. Lin and R. G. Compton, The Journal of Physical Chemistry C, 2015, 119, 2346323469.

40. N. Yoshio, T. Sumiko, G. Moore and P. Moldéus, Biochemical Pharmacology, 1993, 45, 1959-1965.

41. S. S. Batsanov, Inorganic Materials, 2001, 37, 871-885.

42. E. S. C. Kwok and D. A. Eastmond, Chemical Research in Toxicology, 1997, 10, 742749.

43. J. Poon, C. Batchelor-McAuley, K. Tschulik and R. G. Compton, Chem. Sci., 2015, 6, 2869-2876. 